\title{
ROBINS-I: A novel and promising tool for risk of bias assessment of non- randomized studies of interventions
}

\author{
Yasaswi Niranjala Walpita ${ }^{1 *} \&$ Ulugbek Nurmatov $^{2}$ \\ University of Colombo, Sri Lanka; Cardiff University, United Kingdom \\ *Correspondence: yasaswi@commed.cmb.ac.lk \\ https://orcid.org/0000-0001-7608-4042
}

DOI: https://doi.org/10.4038/jccpsl.v26i3.8294

Received on 28 February 2020

Accepted on 27 June 2020

\section{Introduction}

Public health practitioners are often entrusted with the responsibility of evaluating complex communitybased interventions or to compare different health systems, service delivery methods and new policies. For most of these scenarios, it is difficult, unethical, or impossible to conduct randomized trials, so it is inevitable to use Non-Randomized Studies of the effects of Interventions (NRSI) (1-2). The NRSIs are defined as, "any quantitative study estimating the effectiveness of an intervention that does not use randomization to allocate units to intervention groups. Such studies include those in which allocation occurs in the course of usual treatment/practice decisions or according to peoples' choices (i.e. studies often called observational)" (3). Therefore, observational studies such as cohort and case control studies, controlled before-and-after studies, interrupted-time-series studies and quasirandomized studies fall under the category of NRSIs (2-3). In addition, increased use of electronic health records to study large population cohorts using realworld data, also results in many NRSIs within health systems (2).

However, one of the main obstacles in using the evidence synthesized from NRSIs for policy formulation process, is the dilemma of certainty of such evidence due to high chance of a range of potential biases including confounding (1-2, 4). Therefore, it is very important to assess the risk of bias of these studies before using the results for decision making process. In validating research evidence for action, there is a shift in focus around the world from 'assessment of methodological quality and check lists' to 'domain-based risk of bias' (ROB) (2). The tools such as Cochrane ROB tool for randomized trials (5), QUADAS-2 tool for diagnostic test accuracy studies (6) and the ROBIS tool for systematic reviews (7) came into the limelight in this backdrop. Identifying the lack of a tool with similar focus for NRSIs, "Risk Of Bias In Non-randomized Studies of Interventions (ROBINS-I)" tool was developed following a stringent procedure by members of the Cochrane Bias Methods Group and the Cochrane Non-Randomized Studies Methods Group on expert consensus and was substantially revised based on extensive piloting and user feedback $(2,8)$. ROBINS-I was based on Cochrane ROB Tool for randomized trials and related to QUADAS-2 in providing signalling questions, however strictly accustomed to address biases in NRSIs during its development. This tool comprises seven core domains through which bias could be introduced, with up to eight individual points in each domain to be assessed. There is a total of 34 questions. Each domain is rated as 'low', 'moderate', 'serious' or 
'critical' ROB with the overall study rating taking the rating of the worst performing domain. The tool is also accompanied by 53 pages of detailed guidance $(2,8-9)$. ROBINS-I has been available for wider use since the latter part of year 2016 and provides a comprehensive and structured approach for the appraisal of non-randomized studies of interventions.

Quigley et al conducting a review of recommended and commonly used tools for critical appraisal of NRSIs in systematic reviews between 2013-2017, identified 48 different tools being used for critical appraisal of research evidence from NRSIs (9). Table 1 shows a comparison between few common tools identified in this review which further illustrates the focus on domain-based risk of bias assessment in ROBINS-I.

Table 1: Comparison of a few commonly used tools for critical appraisal of NRSIs

\begin{tabular}{|c|c|c|c|c|c|c|c|}
\hline \multirow{2}{*}{ Tool name } & \multirow{2}{*}{$\begin{array}{l}\text { Type of } \\
\text { studies } \\
\text { designed } \\
\text { to assess }\end{array}$} & \multicolumn{5}{|c|}{ Number of items assessing selected domains } & \multirow{2}{*}{$\begin{array}{l}\text { Total no. } \\
\text { of items } \\
\text { in the } \\
\text { tool }\end{array}$} \\
\hline & & $\begin{array}{l}\text { Subject } \\
\text { selection/ } \\
\text { inclusion } \\
\text { criteria }\end{array}$ & Blinding & $\begin{array}{l}\text { Assessment } \\
\text { of outcome/ } \\
\text { exposure }\end{array}$ & $\begin{array}{l}\text { Subject } \\
\text { compar } \\
\text { ability }\end{array}$ & $\begin{array}{l}\text { Confoun } \\
\text { ding }\end{array}$ & \\
\hline ROBINS-I & $\begin{array}{l}\text { NRS of any } \\
\text { intervention }\end{array}$ & 5 & 3 & 10 & - & 8 & 34 \\
\hline MINORS & $\begin{array}{l}\text { NRS of } \\
\text { surgical } \\
\text { interventions }\end{array}$ & 2 & - & 1 & 2 & 1 & 12 \\
\hline NOS & $\begin{array}{l}\text { Case-control } \\
\text { and cohort } \\
\text { studies ( } 2 \\
\text { variations) }\end{array}$ & 4 & - & 3 & 2 & - & 8 \\
\hline \multirow[t]{2}{*}{ SIGN } & $\begin{array}{l}\text { Cohort studies } \\
\text { Case-control }\end{array}$ & 5 & - & 6 & - & 1 & 18 \\
\hline & studies & 6 & - & 2 & - & 1 & 15 \\
\hline
\end{tabular}

ROBINS-I: Risk Of Bias in Nonrandomized Studies of Interventions; MINORS: methodological index for nonrandomized studies; NOS: Newcastle Ottawa Scale; SIGN: Scottish Intercollegiate Guidelines Network; NRS: Non-randomized studies

Though New-Castle Ottawa Scale was commonly used to measure methodological quality of NRSIs, the focus of this tool is not solely on internal validity $(2,10)$. Further, it is also been criticized for the lack of a detailed manuals of application, leads to wide variation in interpretations (interrater reliability) and in terms of the validity of being a scoring system, which in this case rates each awarded "star" equally
(9). In comparison, the review predicted that "ROBINS I tool, may change the future landscape of commonly used NRS critical appraisal tools", due to its superiority over other tools $(2,9,11)$. Key features of ROBINS-I include specification of the target trial and effect of interest, use of signalling questions to inform judgements of risk of bias, and assessments within seven bias domains.

\section{Applications of ROBINS-I}

The ROBINS-I tool was primarily developed for use in the context of a systematic review; however, it has been stated that this could be used to guide 
researchers about issues to consider when designing a primary study to evaluate the effect(s) of an intervention as well as to assess funding applications and peer review of journal submissions (2).

ROBINS-I is mainly aiming at evaluating the risk of bias in estimates of the effectiveness or safety of an intervention, new practice or a policy emanated from studies that did not use randomization (2). Therefore, in application of ROBINS-I, each NRSI is considered as an attempt to mimic a target trial or a hypothetical randomized trial trying to address the same research question addressed by NSRI $(2,11)$. The bias is then defined as a systematic difference between the results of the NRSI and the results expected from the target trial. The tool is based on assessment of seven well defined domains through which bias might be introduced into a NRSI (Figure 1).

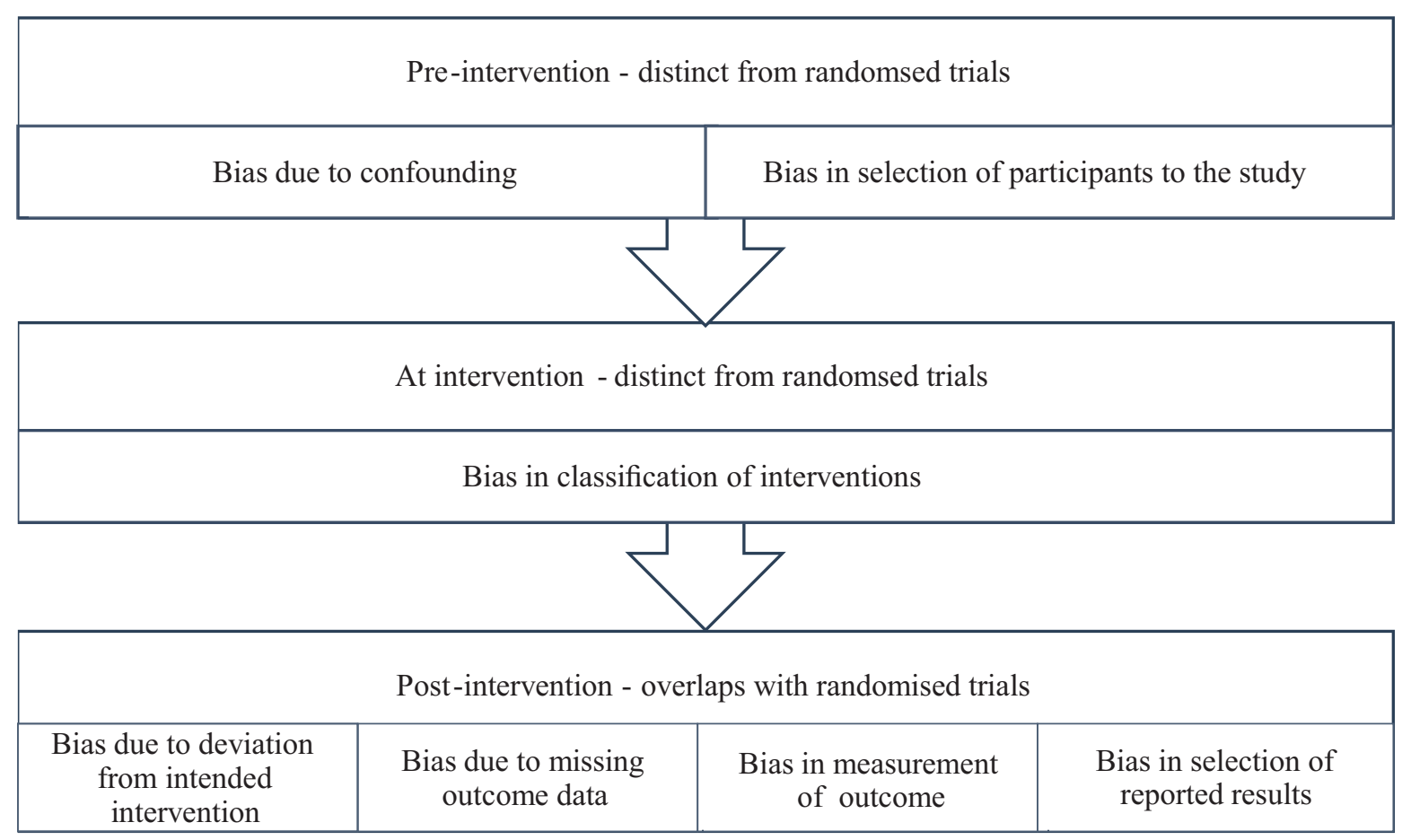

Figure 1: The seven domains of bias assessed in ROBINS-I

The overall risk of bias judgement is categorised as "low risk", "moderate risk", "serious risk" and "critical risk" of bias. The overall judgement is based on the ROB judgements of individual domains. It is worth noting that, "low risk" corresponds to the risk of bias in a high-quality randomized trial, and under exceptional circumstances only a NSRI would be so assessed. The process of applying ROBINS-I to a specific study involves following six steps (Figure 2). 


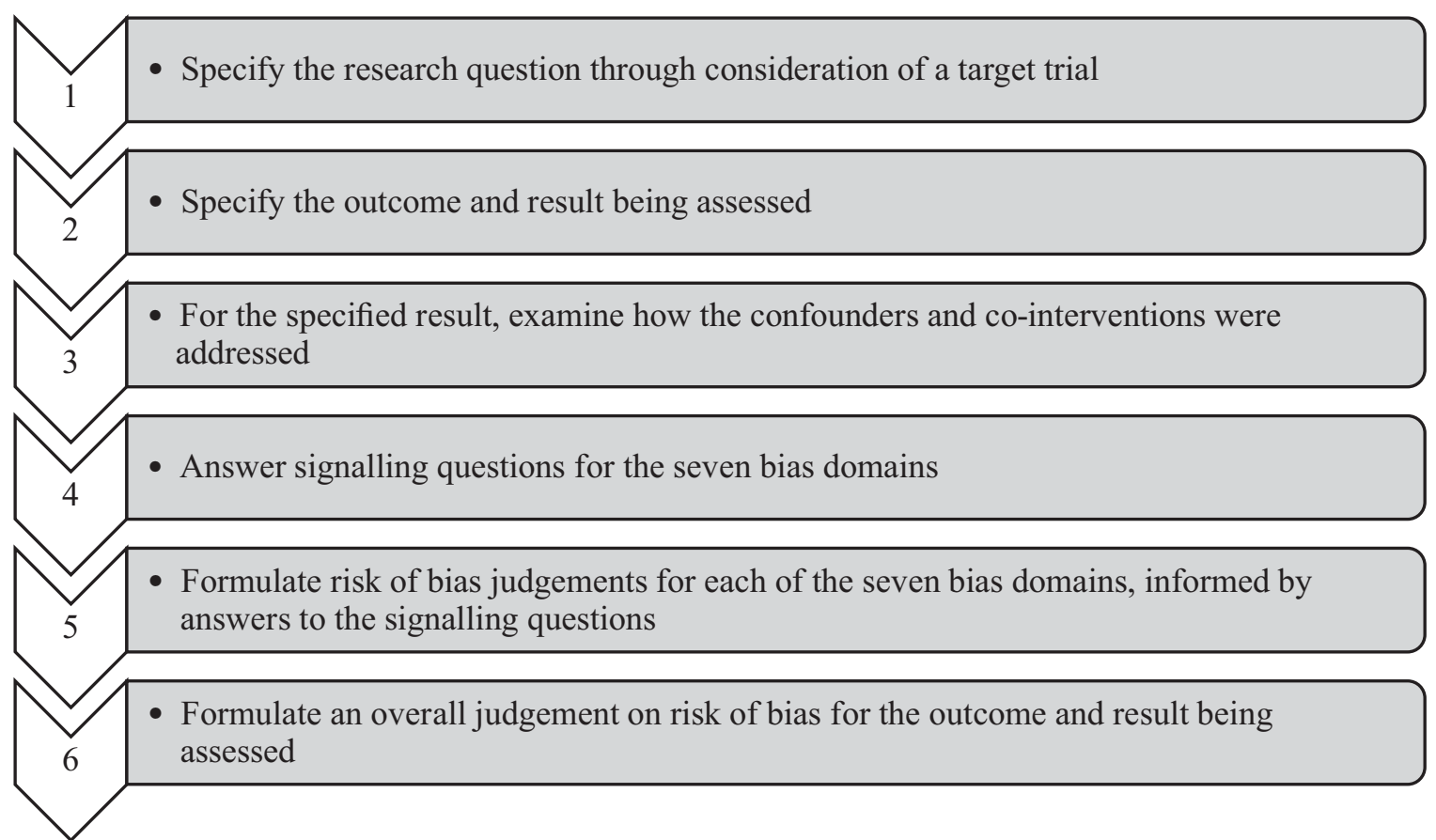

Figure 2: Steps of applying ROBINS-I tool to evaluate a study

\section{Strengths and limitations of ROBINS-I tool}

The most striking feature of this tool is that it is associated with a detailed stepwise guideline (12) on identifying the risk of bias in any study protocol or an already published study through signalling questions. The signalling questions and guidance materials are freely available and downloadable from www. riskofbias.info. Quigley et al, on reviewing the tools used for critical appraisal of NRSIs also expressed promising quality of the then newly introduced ROBINS-I, especially as suitable for any inexperienced reviewer due to welldefined domains and thorough guidance (9).

The Grading of Recommendations Assessment, Development and Evaluation (GRADE) working group, who attempts to advance the certainty rating process of epidemiological studies also claim that, use of ROBINS-I in GRADE approach may allow for a "better comparison of evidence from randomized controlled trials and NRSs because they are placed on a common metric for risk of bias" (11). Further, ROBINS-I is the only tool that explicitly includes a comparison of the relevant NRSI against a 'hypothetical RCT' as of now (11). It also includes an optional component to predict the direction of the bias for each domain, and overall, if deemed necessary to carry out (2). However, it is important to note that, any modifications of the tool are not recommended, even if the context of application differ (2). Concerns also have been raised on the possible misuse of the tool by those wanting to assign a higher certainty to a body of evidence from nonrandomized studies, than is appropriate $(9,11)$. Further, there is still some debate as to whether the results of NRSs and RCTs be considered together in decision making even if the NRSIs were appraised by ROBINS-I (11). When the application of ROBINS-I to natural experiments in public health was studied, the authors expressed difficulties in applying the tool due to the poor reporting and design of such studies (4).

Beside the above limitations ROBINS-I is increasingly recognized world-wide as a conceptually rigorous tool which addresses critical issue of ROB which is not adequately addressed in other quality appraisal tools $(1,11)$. It has also been noted that there will be further refinements to the ROBINS-I tool to address the current limitations too (1-2, personal communication: Jonathan AC Sternae, 5 February 2020). However, only a very few researchers have used this tool yet in the Sri Lankan context. Therefore, it is expected that introducing this 
tool to the wide community of public health practitioners in Sri Lanka, will help in advancing the skills of critical appraisal leading to integration of the best evidence in health care practice.

\section{References}

1. Thomson H, Craig P, Hilton-Boon M, Campbell M, Katikireddi SV. Applying the ROBINS-I tool to natural experiments: an example from public health. Systematic Reviews 2018; 7: 15. doi:10.11 86/s13643-017-0659-4.

2. Sterne JAC, Hernán MA, Reeves BC. et al. ROBINS-I: a tool for assessing risk of bias in nonrandomized studies of interventions. BMJ 2016; 355; i4919. doi:10.1136/bmj.i4919.

3. Reeves BC, Deeks JJ, Higgins JPT. et al. Cochrane Handbook for Systematic Reviews of Interventions. version 6.0. Cochrane Collaboration; 2019. Chapter 24, Including non-randomized studies on intervention effects. Accessed 27 May 2020. Available from: www.training.cochrane.org/ handbook.

4. Craig P, Katikireddi SV, Leyland A, Popham F. Natural experiments: an overview of methods, approaches, and contributions to public health intervention research. Annual Review of Public Health 2017; 38(1):39-56.

5. Higgins JPT, Altman DG, Gøtzsche PC, et al. Cochrane Bias Methods Group Cochrane Statistical Methods Group. The Cochrane Collaboration's tool for assessing risk of bias in randomised trials. $B M J$ 2011;343: d5928. doi:10.1136/bmj.d5928.

6. Whiting PF, Rutjes AW, Westwood ME, et al. QUADAS-2 Group. QUADAS-2: a revised tool for the quality assessment of diagnostic accuracy studies. Annals of Internal Medicine 2011; 155 : 529-36. doi: 10.7326/0003-4819-155-8201110180-00009.

7. Whiting P, Savović J, Higgins JPT, et al. ROBIS Group. ROBIS: A new tool to assess risk of bias in systematic reviews was developed. Journal of Clinical Epidemiology 2016; 69: 225-234. doi: 10.1016/j.jclinepi.2015.06.005.

8. Cochrane methods. Risk of bias tools: risk of bias in non-randomized studies of interventions. Accessed 10 February 2020. Available from: https://www. riskofbias.info/welcome/home.

9. Quigley JM, Thompson JC, Halfpenny NJ, Scott DA. Critical appraisal of nonrandomized studies-A review of recommended and commonly used tools. Journal of Evaluation in Clinical Practice 2019; 25: 44-52.doi:10.1111/jep.12889.

10. Wells GA, Shea B, O'Connell D, et al. The Newcastle-Ottawa Scale (NOS) for assessing the quality of nonrandomised studies in meta-analyses. In 3rd Symposium on Systematic Reviews: Beyond the Basics. Oxford: Ottawa Health Research Institute 2008. Available from: http://www.ohri.ca/ programs/clinical_epidemiology/oxford.asp.

11. Schünemann HJ, Cuello C, Akl EA, et al, GRADE Working Group. GRADE Guidelines: 18. How ROBINS-I and other tools to assess risk of bias in nonrandomized studies should be used to rate the certainty of a body of evidence. Journal of Clinical Epidemiology 2019; 111: 105-114. doi: 10.1016/ j.jclinepi.2018.01.012.

12. Sterne JAC, Higgins JPT, Elbers RG, Reeves BC on behalf of the development group for ROBINS-I. Risk of Bias in Non-randomized Studies of Interventions (ROBINS-I): detailed guidance, updated 12 October 2016. Available from: http:// www.riskofbias.info. 